\title{
EFFECT OF WATER ACTIVITY AND TITANIA P25 PHOTOCATALYST ON INACTIVATION OF PATHOGENIC FUNGI - CONTRIBUTION TO THE PROTECTION OF PUBLIC HEALTH
}

\author{
Agata Markowska-Szczupak ${ }^{1}$, Katarzyna Janda2, Kunlei Wang ${ }^{3}$, Antoni Waldemar Morawski ${ }^{1}$, Ewa Kowalska ${ }^{3,4}$ \\ IInstitute of Inorganic Technology and Environment Engineering, West Pomeranian University of Technology, Szczecin, Poland \\ ${ }^{2}$ Department of Biochemistry and Human Nutrition, Pomeranian Medical University in Szczecin, Szczecin, Poland \\ ${ }^{3}$ Graduate School of Environmental Science, Hokkaido University, Sapporo, Japan \\ ${ }^{4}$ Catalysis Research Centre, Hokkaido University, Sapporo, Japan
}

\section{SUMMARY}

Aim: The aim of this study was to determine the antifungal activity of titanium dioxide activated by indoor light on Czapek Yeast Agar (CYA).

Methods: The agar plate method was used and titania P25 (Evonik) was added to the medium in the amount of $20 \mathrm{~g}_{\mathrm{dm}} \mathrm{dm}^{-3}$. The control experiments without titania were also carried out. The water activity $\left(a_{w}\right)$ of the basal media was adjusted to $0.999,0.997,0.975,0.950,0.900$ and 0.850 with sodium chloride. The photoactivity of titania was tested for six strains of potentially pathogenic fungi: Aspergillus versicolor (WB130, W11), Stachybotrys chartarum (STA1), Pseudallescheria boydii (AP25), Pseudallescheria ellipsoidea (ZUT12), Scedosporium apiospermum (ZUT11), Scedosporium aurantiacum (ZUT4BIS). After inoculation with fungal spore suspensions, one part of CYA plates was incubated in dark, while another part was daily exposed to indoor light for 12 hours per day. The plates were incubated at $25^{\circ} \mathrm{C}$ for 10 days. The daily growth rates $(\mathrm{mm} \times$ day $^{-1}$ ) were calculated from the linear regression equation.

Results: The CYA supplementation with titanium dioxide for different availability of water resulted in the decrease of fungal growth rates in dark and under irradiation. The lowest and the highest rates of daily growth were obtained in the presence and absence of titania for the low and high water activity $\left(0.900 \mathrm{a}_{\mathrm{w}}\right.$ and $\left.0.999 \mathrm{a}_{\mathrm{w}}\right)$, respectively.

Conclusions: Fungi did not grow on all examined media with the lowest water activity $\left(a_{w} \leq 0.850\right)$ which confirmed necessity of water for their growth. The fungi Pseudallescheria-Scedosporium were the most sensitive to titania and water activity, while the strains of Aspergillus versicolor were the most resistant to the presence of titania and lack of water. It has been shown that the lack of accessible water resulted in the strongest action of titanium dioxide both in dark and under irradiation. For the lowest amount of accessible water irradiation of titania resulted in complete inhibition of fungal growth. While, under dark conditions, titania presence enhanced the growth of persistent fungi, i.e., Aspergillus versicolor. It is assumed that these fungi could obtain water from the water-rich titania surface.

Key words: titanium dioxide P25, water activity, pathogenic fungi

Address for correspondence: A. Markowska-Szczupak, Institute of Inorganic Technology and Environment Engineering, West Pomeranian University of Technology, Pulaskiego 10, 70-322 Szczecin, Poland. E-mail: agata@erb.pl

\section{INTRODUCTION}

Microscopic mould fungi are ubiquitous in both indoors and outdoors environments. Approximately 500 fungal species have been described as human pathogens (1). For the recent twenty years an extensive attention has been paid to the potentially pathogenic fungi, which may occur in the indoor environment of flats, offices, schools, kindergartens, etc. It has been noticed that the influence of systematic human exposure to their spores results in increase of allergies, asthma, respiratory problems as well as mycoses in the lungs or on the skin. The infants, children, persons with suppressed immune systems, patients under steroid or chemotherapy treatment are the main groups at risk (2-4). The composition of fungi in the indoor environment depends on various factors, such as temperature, relative humidity, physical and chemical properties of the technical materials, and dust contaminants (3). Because mould grows best in damp and environments damaged by water, e.g. flooded buildings, the water activity of materials may be the most important factor in controlling mildew. The water activity is defined as the ratio of the vapour pressure of water in a material (p) to the vapour pressure of pure water $\left(\mathrm{p}_{\mathrm{o}}\right)$ at the same temperature. Pure distilled water has a water activity of exactly one (5).

People may be exposed to mould either through inhalation or by skin contact with contaminated dust, and thus a certain level of exposure is inevitable. Controlling the level of moisture of technical materials and drying of their surfaces by mechanical, physical and chemical methods can limit microbial growth. Thought, the chemical compounds used for mould inhibition are quite effective for short-term antimicrobial purposes, their application is risky since they are often toxic. In recent years, numerous ecological and environmentally friendly methods have been extensively studied for the removal of various biological pollutants from air, water, wastewater and surfaces of technical materials. Among them photocatalytic oxidation on titanium dioxide is one of the best known. 
$\mathrm{TiO}_{2}$ has been widely used for water disinfection, wastewater treatment, air deodorization, self-cleaning and self-sterilizing surfaces $(6,7)$. However, the majority of studies on the antimicrobial activity of titanium dioxide were performed for bacteria and yeast (8-10). There are only a few studies focusing on the photocatalytic removal of potentially pathogenic fungi since they are much more persistent than simple bacteria organisms (11-13).

The aim of the study was to examine the influence of titania (titanium dioxide, $\mathrm{TiO}_{2}$ ) activated by indoor light and water activity on few species of potentially pathogenic fungi. Aspergillus $s p$. is genus consisting of several hundred species found in various climate zones. A. versicolor, A. flavus and A. fumigatus are frequently found in the food products such as stored cereals, hay, cheese, meat etc. A. fumigatus has secreted secondary metabolites (mycotoxins) such as 6-methoxysterigmatocystine and aversine, and has been also reported as a causative agent of cutaneous disease, bilateral toenail onychomycosis, osteomyelitis and many others (14). Black mould Stachybotrys chartarum is primarily isolated from cellulose-rich building materials (such as wallpaper, wood etc.) in damp and water-damaged buildings. Stachybotrys chartarum secrets neurotoxin, i.e., stachratoxin H, and is often connected with the Sick Building Syndrome (SBS) defined as the combination of symptoms associated with an individual's place of work or residence (15). Pseudallescheria boydii is a complex of species, e.g., Ascomycetes and also their products, such as asexual anamorphs, mainly Scedosporium. Species of Pseudallescheria sp. and Scedosporium sp. cause human scedosporiosis, a fungal disease difficult to treat with a high mortality rate $(16,17)$.

\section{MATERIALS AND METHODS}

The antifungal activity of $\mathrm{TiO}_{2}$ and water was investigated on standard Czapek Yeast Agar (CYA, BTL) using agar plate method. The basal medium was supplemented with $20 \mathrm{~g} \cdot \mathrm{dm}^{-3}$ of $\mathrm{TiO}_{2}$ (P25 from Evonik company, Germany). P25 titania is one of the most active titania photocatalyst (18), used worldwide in various areas, e.g., water or wastewater purification, air treatment, self-cleaning and antifogging surfaces. The exemplary characteristic of titanium dioxide P25, i.e., its crystalline properties (X-ray crystallography, XRD), photoabsorption properties (diffuse re- flectance spectroscopy, DRS) and morphology (scanning electron microscopy, SEM), is presented in Fig. 1. XRD pattern clearly shows that P25 is a mixture of two crystalline phases of titania, i.e., anatase (main part) and rutile. It has already been reported that content of both phases varied for different $\mathrm{P} 25$ samples reaching 73-85 wt $\%$ and $14-17 \mathrm{wt} \%$ for anatase and rutile, respectively (18). P25 consists also of amorphous titania in the amount of $1-13 \mathrm{wt} \%$ (data obtained from XRD analysis of P25 mixed with highly crystalline $\mathrm{NiO}$ used as internal standard). P25 is able to absorb light at UV and visible range till ca. $420 \mathrm{~nm}$, due to rutile presence which possesses narrower band-gap than anatase (ca. $3.0 \mathrm{eV}$ and $3.2 \mathrm{eV}$, respectively). The XRD calculated crystalline sizes of anatase and rutile of $28.1 \mathrm{~nm}$ and $36.5 \mathrm{~nm}$ (average data), respectively, indicate that smaller crystallites observed in SEM image are due to anatase presence. It should be mentioned that presented data are exemplary, and P25 is highly heterogenic material (18), and even samples taken from the same container slightly differ in composition.

The water activity of the media was adjusted with sodium chloride to $\mathrm{a}_{\mathrm{w}}$ : $0.999,0.997,0.975,0.950,0.900$ and 0.850 (19). The $\mathrm{a}_{\mathrm{w}}$ was confirmed with a DE 202 Aqua Lite meter. The control experiments without $\mathrm{TiO}_{2}$ and sodium chloride were also performed.

The $\mathrm{TiO}_{2}$ antifungal effectiveness was examined using three strains isolated from air in water-damaged building's cellar: Aspergillus versicolor (strains: WB130, WB11), Stachybotrys chartarum (STA1). Four strains of Pseudallescheria-Scedosporium complex fungi obtained from hospital: Pseudallescheria boydii (AP25), P. ellipsoidea (ZUT12), Scedosporium apiospermum (ZUT11), Scedosporium aurantiacum (ZUT4BIS) were isolated from patients (throat smear). Ten days old fungal cultures grown on Malt Extract Agar (MEA) slants at $25^{\circ} \mathrm{C}$ were used for preparation of spore suspensions. $5 \mathrm{ml}$ of sterile physiological saline were added to each slant. The slants were then vigorously shaken by a vortex for 3 minutes. Each plate was centrally inoculated with 5 $\mu \mathrm{l}$ of spore suspension. One part of the plates was incubated in dark, while another part was exposed to indoor light. The natural indoor light (intensity ca. $120 \mathrm{~W} \cdot \mathrm{m}^{-2}$ ) was strengthened with 8-hour daily exposition to halogen light (intensity ca. $130 \mathrm{~W} \cdot \mathrm{m}^{-2}$ ). The distance from three halogen lamps $(50 \mathrm{~W}$ each) to the plates was ca. $50 \mathrm{~cm}$. The participation of UV range in overall irradiation by halogen lamp was very low since UV intensity did not exceed ca.

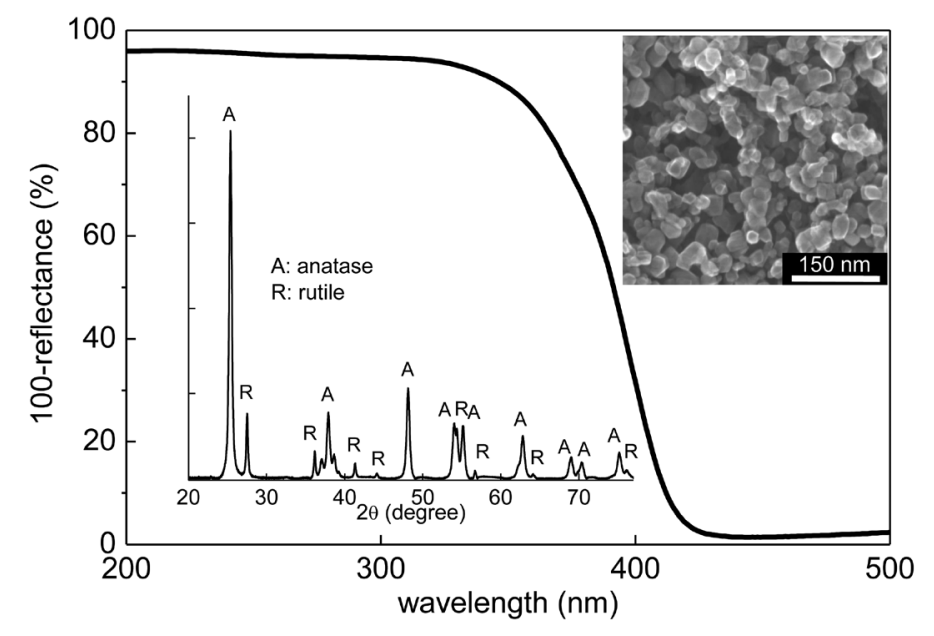

Fig. 1. Exemplary characteristic of titania P25 photocatalyst: (centre) photoabsorption properties (DRS spectrum), (left) XRD pattern, (right) SEM image. 
$0.3 \mathrm{~W} . \mathrm{m}^{-2}$. The incubation was carried out for 10 days at $25^{\circ} \mathrm{C}$. The colony diameters were measured after 2, 4, 6, 8 and 10 days of incubation. The daily growth rates $(\mathrm{mm} /$ day) were calculated from the linear regression equation, $r=a \cdot t+b$, where: $r$ - colony radius $(\mathrm{mm}), \mathrm{t}$ - incubation time (day), a - daily growth rate, and $\mathrm{b}$ - growth retardation time (lag phase; $\lambda$ ). According to Dantigny et al. (20), the lag time (the time from inoculation of fungal spores to growth phase of fungi) has no biological significance, because it is calculated from macroscopic observations of mycelium. Thus, the $b$ values were abandoned in this study.

\section{RESULTS AND DISCUSSION}

The inhibition effect of titanium dioxide on fungal growth in dark was observed in all examined Pseudallescheria complex fungi and Stachybotrys chartarum for various water activities, Aspergillus versicolor $\mathrm{W} 130$ and $\mathrm{WB} 11$ for $\mathrm{a}_{\mathrm{w}} \geq 0.975$, A. versicolor ZUT25 for $\mathrm{a}_{\mathrm{w}} \geq 0.950$. Almost all differences were statistically significant at $\mathrm{p} \leq 0.05$. Subsequently, the $\mathrm{TiO}_{2}$ enhanced fungal growth for $A$. versicolor $\mathrm{W} 130$ and $\mathrm{WB} 11$ for $\mathrm{a}_{\mathrm{w}} \leq 0.950$ and A. versicolor ZUT25 for $\mathrm{a}_{\mathrm{w}} \leq 0.900$ (Fig. 2). Thus, the fungal reaction toward titanium dioxide was almost independent on place of fungal strain isolation (the similar behaviour was noticed for three strains). The highest inhibition of fungal growth was observed for Pseudallescheria-Scedosporium complex fungi (Fig. 3), probably due to the high sensitiveness of these fungi towards many antifungal agents (17). These fungi possess a very unique composition of glycopeptides, which are important determinants for the cell response to environmental stimuli and biological activities. It is thought that titanium dioxide weakens the cell walls, making them fragile and unable to grow. These results confirm that the presence of $\mathrm{TiO}_{2}$ particles in media influence the fungal growth on $\mathrm{TiO}_{2}$ surface as has already been proposed by Pichat (21). However, based on the available literature it is impossible to explain the stimulative effect of titanium dioxide on fungi (6). Here, for the first time, it is proposed that in the case of low water activity, titania could provide water supply for fungal growth since it may contain up to a few percent of physisorbed water, e.g., 2-3\% of water was detected by thermogravimetric (TGD) analysis for commercial P25 (22).

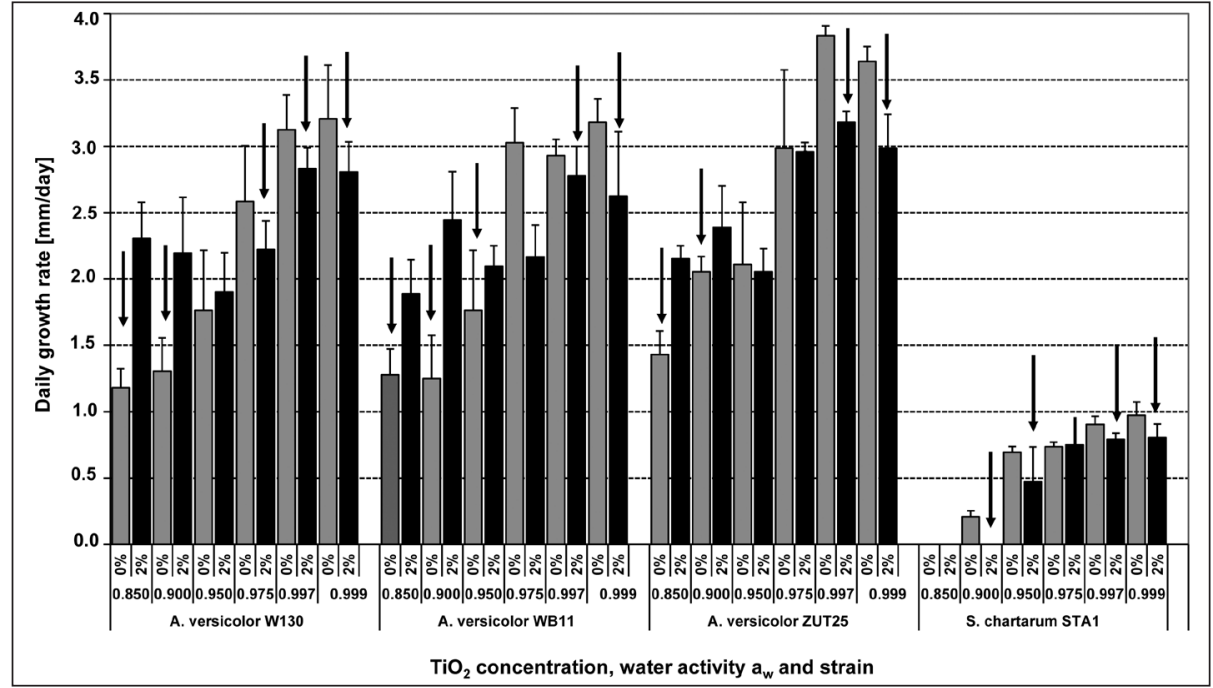

*Black bars mean standard deviations between replications.

${ }^{* *}$ Black arrows mean statistically significant differences between daily growth rate on control media and media with $\mathrm{TiO}_{2}(\%)$ at $p \leq 0.05$.

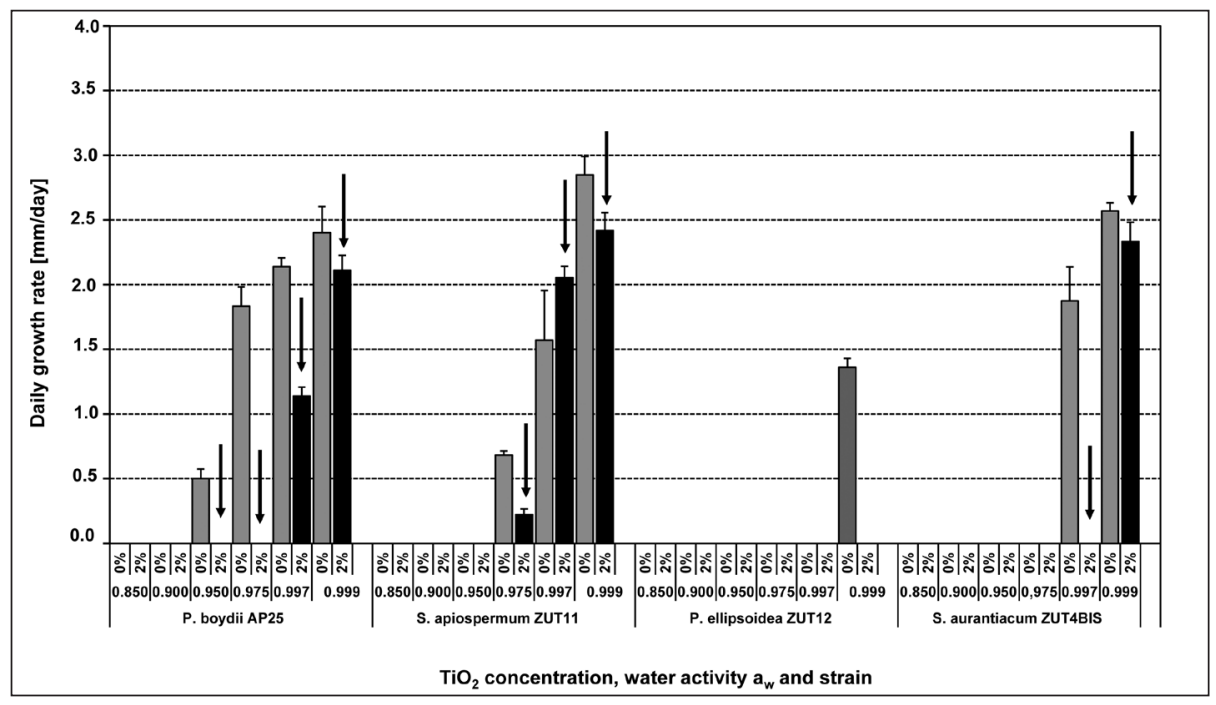

Fig. 3. Mean daily radius growth of strains of Pseudallescheria-Scedosporium complex fungi on media with different water activity and concentration of $\mathrm{TiO}_{2}(\%)$, incubated in dark conditions.

${ }^{* *}$ Black arrows mean statistically significant differences between daily growth rate on control media and media with $\mathrm{TiO}_{2}(\%)$ at $\mathrm{p} \leq 0.05$. 


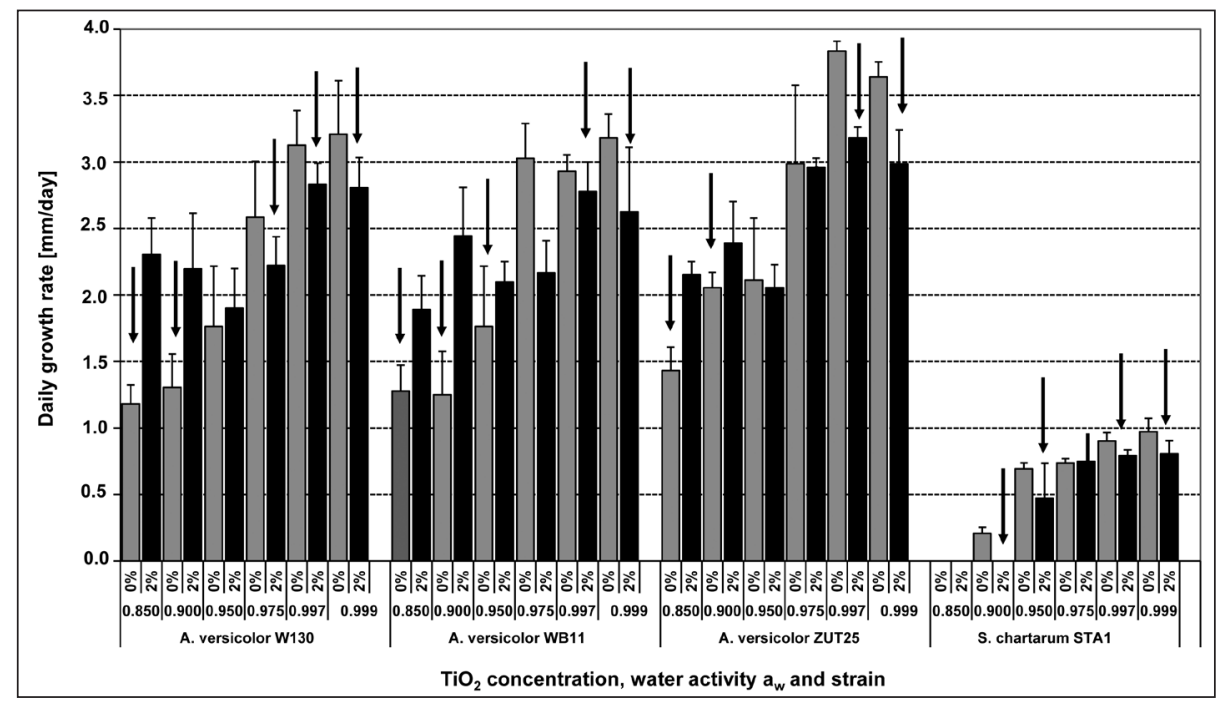

*Black bars mean standard deviations between replications.

**B Black arrows mean statistically significant differences between daily growth rate on control media and media with $\mathrm{TiO}_{2}(\%)$ at $p \leq 0.05$.
Fig. 4. Mean daily radius growth of strains of Aspergillus versicolor and Stachybotrys chartarum on media with different water activity and concentration of $\mathrm{TiO}_{2}(\%)$, incubated in light conditions.

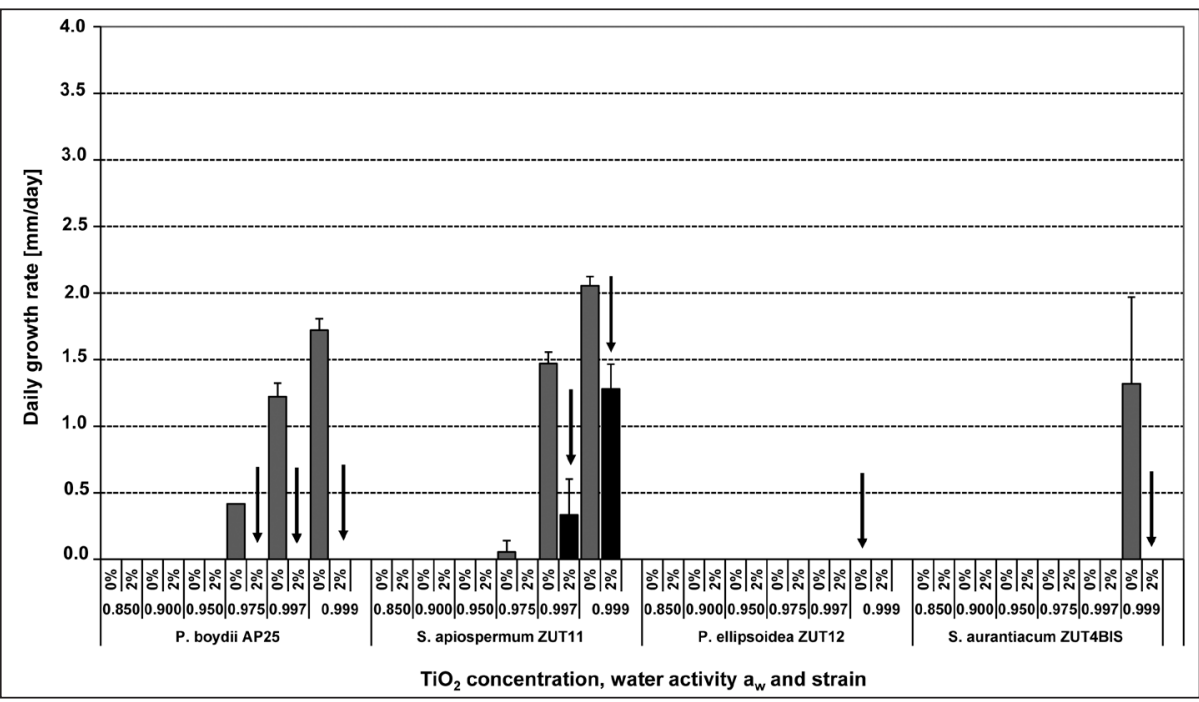

Fig. 5. Mean daily radius growth of strains of Pseudallescheria-Scedosporium complex fungi on media with different water activity and concentration of $\mathrm{TiO}_{2}(\%)$, incubated in light conditions.

*Black bars mean standard deviations between replications.

${ }^{* *}$ Black arrows mean statistically significant differences between daily growth rate on control media and media with $\mathrm{TiO}_{2}(\%)$ at $\mathrm{p} \leq 0.05$.

The indoor light mostly constrained fungal growth and completely inhibited the growth of P. ellipsoidea (Figs. 3, 5). All examined fungi did not grow on CYA media supplemented with titanium dioxide when water activity was equal to 0.850 (Figs. $2-5$ ). The explanation of aberrant response of fungi was postulated by Kakde and Chavan (23) as destructive mechanism of light action. Light plays an important role in the inhibition of enzymes and metabolite secretion in fungal cells. The action of light on fungal growth can be obtained by comparison of results shown in Figures 2 and 4, and Figures 3 and 5. It is clearly demonstrated that single light (in the absence of titania) inhibited the fungal growth, especially at low water activity, e.g., complete inhibition of fungal growth was observed under irradiation of $S$. chartarum and $P$. boydii, at 0.900 and $0.950 \mathrm{a}_{\mathrm{w}}$, respectively. The inhibition of fungal growth was also observed at higher water activity, e.g., ca. 80 and $90 \%$ inhibition of fungal growth was achieved at 0.975 $\mathrm{a}_{\mathrm{w}}$ for P. boydi and S. Apiospermum, respectively.

Though, light, temperature, $\mathrm{pH}$ and several other factors affect organism growth, the water activity may be the most important factor in controlling spoilage. A lower water activity inhibits the fungal spore germination and mould proliferation (24). The fungal growth was inhibited the most when samples were irradiated in the presence of $\mathrm{TiO}_{2}$ photocatalysts for low water activity. The action of irradiated titania can result from direct reaction with photo-generated charge carriers (electrons and holes), or oxidative decomposition of fungal cells by generated ROS (reactive oxygen species), such as hydrogen peroxide, singlet oxygen, superoxide anion radical. The low enhancement of activity after photocatalyst addition for high water activity is still unclear and could be caused by super-excessive growth of fungi in these "wet" conditions, and relatively low activity of $\mathrm{TiO}_{2}$ under indoor irradiation of very low UV intensity (necessary for $\mathrm{TiO}_{2}$ activation, band gap of ca. $3 \mathrm{eV}$ ). It is thought that application of more intense light sources, such as UV lamps, should result in complete inhibition of fungal growth. However, in the present study UV lamps have been avoided due to necessity of development of environmental friendly materials able to work under natural solar radiation. Our recent research on Penicillum chrysogenum has proved that modification of titania with nanoparticles of noble metals being able to absorb visible light (due to plasmon resonance) caused 
inhibition of both fungal growth and production of mycotoxins under irradiation with visible light (25).

The activity of titanium dioxide was observed in almost all cases (Figs. 2-5). It was found that the strains of the Pseudallescheria boydii complex and the Stachybotrys chartarum strain were the most sensitive to titanium dioxide and low amount of water when incubated under light (Figs. 4, 5), while the Aspergillus versicolor strains were the most resistant to these factors (Fig. 4).

It was demonstrated that lower water activity causes higher action of titanium dioxide under both dark and light (Figs. 2-5). As it was presented in the previous studies (13), a considerable impact of indoor light-activated $\mathrm{TiO}_{2}$ on media with different water activity was observed for the first 2-4 days of incubation.

Our study has shown that the concentration of $20 \mathrm{~g}$ of $\mathrm{TiO}_{2}$ per $\mathrm{dm}^{3}$ is not sufficient for the elimination of Aspergillus versicolor and Stachybotrys chartarum. Similar observation has been already reported for Aspergillus niger by Chen, et al. (12), i.e., that these species are generally resistant to the photocatalytic process also under strong irradiation with UVA. In addition, many Aspergilli species have been described as a fungus resistant for lower water activity, i.e., they are xerophilic fungi. Xerophiles are more common in high dry environments with water activity bellow 0.800 (26).

\section{CONCLUSION}

Although our results on agar medium may not exactly represent the real germination and mycelial growth capacity on technical materials or walls under fluctuating temperature and moisture levels, they could provide an approximation of conditions favourable for development of frequently occurring pathogenic fungi.

At present, further studies on direct correlation between properties of $\mathrm{TiO}_{2}$, water activity of technical materials and indoor light are carried out. The necessity of development of active materials able to inhibit fungal growth is still urgent and should be investigated. These studies ought to be focused on finding out more efficient materials with ability of working under natural solar radiation, and to explain the role of crystalline forms, i.e., anatase and rutile, and non-crystalline phase in the mechanisms of fungi killing by $\mathrm{TiO}_{2}$. Obtained experimental data should allow the chemical technologist to design an indoor photocatalytic paints and coatings being able to effectively decrease the growth of potentially pathogenic fungi occurring in the human environment under natural conditions, i.e., scattered daylight.

\section{Acknowledgement}

This study was financially supported by the National Centre for Science No. DEC-2012/06/A/ST5/00226 and by Bill \& Melinda Gates Foundation through the Grand Challenges Exploration Initiative (OPP1060234).

\section{Conflict of Interests}

None declared

\section{REFERENCES}

1. Kirk PM, Cannon PF, Minter DW, Stalpers JA. Dictionary of the Fungi. 10th ed. Wallingford: CAB International; 2008
2. Sivasubramani SK, Niemeier TT, Reponen T, Grinshpun SA. Assessment of the aerosolization potential for fungal spores in moldy homes. Indoor Air. Indoor Air. 2004 Dec;14(6):405-12.

3. Gutarowska B. Metabolic activity of moulds as a factor of building materials biodegradation. Pol J Microbiol. 2010 Apr 8;59(2):119-24.

4. Mitchell CS, Zhang JJ, Sigsgaard T, Jantunen M, Lioy PJ, Samson R, et al. Current state of the science: health effects and indoor environment. Environ Health Perspect. 2007 Jun;115(6):958-64.

5. Laroche C, Fine F, Gervais P. Water activity affects heat resistance of microorganisms in food powders. Int J Food Microbiol. 2005 Jan 1;97(3):307-15.

6. Markowska-Szczupak A, Ulfig K, Morawski AW. The application of titanium dioxide for deactivation of bioparticulates: an overview. Catal Today. 2011 Jul 1;169(1):249-57.

7. Paz Y. Application of $\mathrm{TiO}_{2}$ photocatalysis for air treatment: patents overview. Appl Cat B: Environ. 2010 Sep 9;99(3-4):448-60.

8. Malato S, Fernández-Ibáñez P, Maldonado MI, Blanco J, Gernjak W. Decontamination and disinfection of water by solar photocatalysis: recent overview and trends. Catal Today. 2009 Sep 15;147(1):1-59.

9. Piegot-Rémy S, Simonet F, Errazuriz-Carda E, Lazzaroni JC, Atlan D, Guillard C. Photocatalysis and disinfection of water: identification of potential bacterial target. Appl Cat B: Environ. 2011 May 18;104(3-4):390-8.

10. Coleman HM, Marquis CP, Scott JA, Chin SS, Amal R. Bactericidal effects of titanium dioxide-based photocatalysts. Chem Engin J. 2005 Oct 1;113(1):55-63.

11. Hochmannova L, Vytrasova J. Photocatalytic and antimicrobial effects of interior paint. Progr Organic Coat. 2010 Jan;67(1):1-5.

12. Chen F, Yang X, Wu Q. Antifungal capability of $\mathrm{TiO}_{2}$ coated film on moist wood. Build Environ. 2009 May;44(5):1088-93.

13. Markowska-Szczupak A, Ulfig K, Morawski AW. Antifungal effect of titanium dioxide, indoor light and the photocatalytic process in in vitro test on different media. J Adv Oxid Technol. 2012 Jan;15(1):30-3.

14. Torres-Rodríguez JM, Madrenys-Brunet N, Siddat M, López-Jodra O, Jimenez T. Aspergillus versicolor as cause of onychomycosis: report of 12 cases and susceptibility testing to antifungal drugs. J Eur Acad Dermatol Venereol. 1998 Jul;11(1):25-31.

15. Andersen B, Nielsen KF, Jarvis BB. Characterization of Stachybotrys from water-damaged buildings based on morphology, growth, and metabolite production. Mycologia, 2002 May-Jun;94(3):392-403.

16. Gilgado F, Cano J, Gené J, Guarro J. Molecular phylogeny of the Pseudallescheria boydii species complex: proposal of two new species. J Clin Microbiol. 2005 Oct;43(10):4930-42.

17. Lopes LC, Rollin-Pinheiro R, Guimarães AJ, Bittencourt VCB, Martinez LR, Koba W, et al. Monoclonal antibodies against peptidorhamnomannans of Scedosporium apiospermum enhance the pathogenicity of the fungus. PLoS Negl Trop Dis. 2010 Oct 19;4(10):e853.

18. Ohtani B, Prieto-Mahaney OO, Li D, Abe R. What is Degussa (Evonik) $\mathrm{P} 25$ ? Crystalline composition analysis, reconstruction from isolated pure particles and photocatalytic activity test. J Photochem Photobiol A: Chem. 2010 Dec 15;216(2-3):179-82.

19. Mazurkiewicz L, Tomasik P, Zapłotny J. Relationships between water activity and viscosity of solutions. Food Hydrocoll. 2001 Jan;15(1):43-6.

20. Dantigny P, Guilmart A, Bensoussan M. Basis of predictive mycology. Int J Food Microbiol. 2001 Apr 15;100(1-3):187-96.

21. Pichat P. A brief survey of the potential health risks of $\mathrm{TiO}_{2}$ particles and $\mathrm{TiO}_{2}$ containing photocatalytic or non-photocatalytic materials. J Adv Oxid Technol. 2010 Jul;13(3):238-46.

22. Wang K. Study on photocatalytic activity of anatase-rutile titania particles isolated from commercial Evonik P25. MSc thesis. Hokkaido: Hokkaido University; 2014.

23. Kakde RB, Chavan AM. Extracelluar lipase enzyme production by seed-borne fungi under the influence of physical factors. Int J Biol. 2011 Jan;3(1):94-100.

24. Rosso L, Robinson TP. A cardinal model to describe the effect of water activity on the growth of moulds. Int J Food Microbiol. 2001 Feb 15;63(3):265-73.

25. Kowalska E, Wei Z, Karabiyik B, Janczarek M, Endo M, Wang K, et al. Development of plasmonic photocatalysts for environmental application. Adv Sci Technol. 2014 Oct;93:174-183.

26. Gock MA, Hocking AD, Pitt JI, Poulos PG. Influence of temperature, water activity and $\mathrm{pH}$ on growth of some xerophilic fungi. Int J Food Microbiol. 2003 Feb 25;81(1):11-9. 